\title{
Fibrillin-1 (FBN1) gene frameshift mutations in Marfan patients: genotype-phenotype correlation
}

Pepe G, Giusti B, Evangelisti L, Porciani MC, Brunelli T, Giurlani L, Attanasio M, Fattori R, Bagni C, Comeglio P, Abbate R, Gensini GF. Fibrillin-1 $(F B N 1)$ gene frameshift mutations in Marfan patients: genotype-phenotype correlation.

Clin Gene 2001: 59: 440-450. (C) Munksgaard, 2001

Marfan syndrome (MFS) is a multisystemic disease associated with mutations in the fibrillin-1 gene. Most of the reported mutations are missense substitutions mainly affecting the epidermal growth factor (EGF)-like protein domain structure and the calcium-binding (cb) site. The aim of our study was to investigate the correlation between fibrillin-1 frameshift mutations and the clinical phenotype in patients affected by MFS. In 48 out of 66 Marfan patients a pathogenetic mutation was found. We detected novel mutations causing premature termination codon in exons $19,37,40$ and 41 of four Italian patients. The first mutation in exon 19 (cbEGF \# 8 domain) results in a clinical phenotype involving mainly the skeletal and cardiovascular systems. Interestingly, we noticed that, while mutations in exons 37 and 41 (eight cysteine domains \# 4 and \# 5) are milder, the mutation in exon 40 (cbEGF \# 24 domain) is more severe and causes major cardiovascular involvement with thoracic and abdominal aortic aneurysms. It is noteworthy that the degree of the severity in the phenotype of one of our patients and another from the literature carrying a mutation in exon 41 could be explained with alterations in mRNA expression.

\author{
G Pepe ${ }^{a, b}$, B Giustia, \\ L Evangelisti ${ }^{a}$, MC Porciani ${ }^{a}$, \\ T Brunellia, L Giurlania, \\ M Attanasio ${ }^{a}$, R Fattori, \\ C Bagnic, P Comeglio ${ }^{a}$, \\ R Abbate ${ }^{a}$ and GF Gensinia \\ a Dipartimento di Area Critica Medico \\ Chirurgica, sezione Clinica Medica Generale \\ e Cliniche Specialistiche, University of \\ Florence, Florence, ${ }^{b}$ Dipartimento di \\ Medicina Interna $e^{\mathrm{c}}$ Dipartimento di \\ Biologia, University of Rome 'Tor Vergata', \\ Rome and 'Dipartimento di Radiologia, \\ Unità Cardiovascola, University of Bologna, \\ Bologna, Italy
}

Key words: aortic aneurysm - fibrillin-1 gene - frameshift mutations - Marfan syndrome - MASS

Corresponding author: Guglielmina Pepe, MD, Dipartimento di Area Critica Medico Chirurgica, sezione Clinica Medica Generale e Cliniche Specialistiche, University of Florence, Florence, Viale Morgagni 85, 50134, Florence, Italy. Tel: + 39055 4279417; fax: + 39055 4279418; e-mail: labctrombosi@ao-careggi.toscana.it

Received 13 December 2000, revised and accepted for publication 5 March 2001
Marfan syndrome (MFS) is a pleiotropic inherited connective tissue disorder with autosomal dominant transmission and a prevalence of $1 / 5000$ (1). This disease involves the cardiovascular, skeletal, ocular, and central nervous systems, lungs, skin and integument (2). For MFS, major criteria (more severe clinical symptoms) affecting at least two organ systems and the involvement of a third with minor criteria (milder clinical symptoms) are required. A first degree relative with MFS also constitutes a major criterion (3).

Fibrillin-1 (FBN1) is the gene linked to MFS (4) in at least $80 \%$ of the cases (5). This gene encodes an extracellular matrix glycoprotein, which is one of the main constituents of the $10 \mathrm{~nm}$ microfibrils and has two functions: a) it represents the basal network for tropoelastin growth in elastic tissues and b) it acts as an anchoring structure in nonelastic tissues $(6,7)$. The $F B N 1$ gene is about 110 $\mathrm{kb}$ long while the cDNA, made up of 65 exons, is approximately $10 \mathrm{~kb}$. The protein is mainly made up of calcium-binding epidermal growth factor $(\mathrm{cbEGF})$-like $(\mathrm{n}=43)$, EGF-like $(\mathrm{n}=4)$ and cysteine rich $(\mathrm{n}=7)$ domains interacting with many extracellular matrix components $(8,9)$.

At present, more than 135 mutations in FBN1 have been characterized. Most of them were found in classic MFS patients, several in neonatal Marfans and a few in patients affected by ectopia lentis, MASS (M = myopia and mitral valve pro- 
lapse; $\mathrm{A}=$ aortic dilation; $\mathrm{S}=$ skeleton; $\mathrm{S}=$ skin) and Shprintzen-Goldberg syndrome. Mutations in the $F B N 1$ gene were also identified in other Marfanrelated disorders (9). The 11 frameshift deletion/insertion mutations $(<20 \mathrm{nts})$ creating a premature termination codon (PTC) amount to less than 10\% of the total mutations and were detected in 10 Marfan patients, 4 of whom are classified as clinically mild (5, 9-11) and 1 MASS patient (12).

In the present study, we report the molecular characterization of four novel frameshift mutations in Italian patients, all affected by classic MFS. We compare our data with those of the literature and correlate the genotype with the clinical phenotype. All of the frameshift insertions/deletions so far detected in FBN1 are also reviewed showing that they are probably a result of slippage mispairing at the replication fork $(13,14)$. These frameshift mutations were characterized during a wider mutation detection study involving 66 Marfan patients, in which mutations in FBN1 were found in 48 of 66 patients $(72.7 \%)$.

\section{Materials and methods}

Subjects

The patients came to our attention at the Marfan Clinical and Research Center of the University of Florence. Family history and medical records were collected by four of us (G.P., M.C.P., L.G. and R.F.). All patients underwent a multidisciplinary (cardiologist, internist, ophthalmologist, orthopedic, clinical geneticist) clinical examination. The diagnosis of classic Marfan phenotype was made according to international diagnostic criteria (3). The 4 patients with frameshift mutations specifically described in this paper are referred to as $\mathrm{P}$ (patient) 4 (S.B.), P5 (E.P.), P6 (A.M.) and P7 (G.B.).

Magnetic resonance imaging (MRI) of the lumbo-sacral spine was performed and the degree of dural alterations was classified as reported by Fattori et al. (15) from 0 (normal dural sac) to 3 (anterior sacral meningocele).

\section{DNA isolation}

Genomic DNA was extracted from patients and controls from peripheral blood as already described by Sambrook et al. (16). Informed consent was obtained from the subjects and their families.

\section{Conformational Strand Gradient Electrophoresis (CSGE)}

Heteroduplex analysis was carried out according to Ganguly et al. $(17,18)$ as previously described (19).
The 65 FBN1 exons were amplified separately by polymerase chain reaction (PCR), using synthetic oligonucleotides as described by Nijbroek et al. (5).

\section{Direct sequencing}

Whenever a heteroduplex was detected, direct sequencing analysis of the amplified exons was performed using the same oligonucleotides utilized for the PCR as primers according to Pepe (20). By loading an aliquot of the PCR amplified DNA fragments corresponding to exons 19, 37, 40 and 41 and containing the mutations on a denaturing polyacrylamide gel, we were able to separate the two alleles of the DNA of each patient (data not shown). Otherwise, we performed an SSCP (single strand conformation polymorphism) analysis to collect the mutant homozygous bands (21). The single alleles $(1-5 \mu$ of the elution) were reamplified and directly sequenced.

RNA extraction and reverse transcriptase-polymerase chain reaction (RT-PCR)

Total RNA was prepared from confluent fibroblast cells in culture of P5 using the RNA fast kit (Biotecx Laboratories Inc., Houston, TX, USA) and treated with reverse transcriptase to obtain a pool of $\mathrm{cD}$ NAs, as described by Attanasio et al. (22). The cDNA region containing the FBN1 exon 417 nts deletion was co-amplified with the wild type allele by PCR with the following oligonucleotides:

\section{CF10S 5'-CGTTGGCAACTACACCTGTA-3'}

and

\section{CF10AS 5'-TTACAGTCACAGCGGTAGCT-3'.}

Two fragments of 502 and $509 \mathrm{nt}$ were separated on a $6 \%$ denaturing acrylamide gel and visualized by ethidium bromide staining.

Semi-quantitative RT-PCR analysis of mutant FBN1 mRNA

Hot RT-PCR amplification of total RNA with a $5^{\prime}$ kinase $\gamma$-ATP- ${ }^{33}$ P-labeled oligonucleotide (CF 10s) was carried out at different cycles $(17,20,23,26,29)$ as already reported (23) and the two alleles were separated as described above. The dried gel underwent autoradiography and the impressed film was analyzed by a densitometric scanning.

\section{Homocysteine}

In all patients, total homocysteine plasma levels were determined by HPLC with fluorimetric detection according to Araki and Sako (24). 


\section{Results}

Of 66 patients that undergo CSGE analysis and direct sequencing, 48 displayed heteroduplexes and 4 of them carried a deletion/insertion pattern. P4, P5, P6 and P7 showed extra bands with respect to controls in exons 37, 41, 40 and 19, respectively (data not shown). P6 and P7 displayed a severe/ mild phenotype while P4 and P5 are moderately affected.

All the frameshift mutations characterized in this study are reported in Table 1 .

\section{Clinical histories and molecular analysis}

P6 is a 27-year-old Caucasian male (height $204 \mathrm{~cm}$ ) with sporadic infantile MFS $(19,25)$ involving the ocular, skeletal, central nervous and cardiovascular systems. He presented with myopia since the age of
13 and blue sclerae. Positive wrist and thumb signs, pes planus, pectus excavatum requiring surgery, arched palate with crowding of the teeth, retrognathia, dolichostenomelia, scoliosis and arachnodactyly were evident. Joint hypermobility of both shoulders and knees and two episodes of spontaneous pneumothorax between the age of 10 and 11 were reported. Striae atrophicae not associated with marked weight changes were observed. MRI of the aorta documented the presence of aortic root dilatation at the sinuses of Valsalva (48 $\mathrm{mm} ; 2.41 \mathrm{~cm} / \mathrm{m}^{2}$ body surface area), mitral valve prolapse and an abdominal aortic aneurysm (AAA) which was discovered when he was 6 years old. Furthermore, MRI of the lumbo-sacral region showed the presence of dural ectasia (MRI of grade 2). His brother (31 years old; height $188 \mathrm{~cm}$ ) presented with mild involvement of the ocular and

Table 1. Insertion/deletion (Ins/Del) frameshift mutations causing PTC

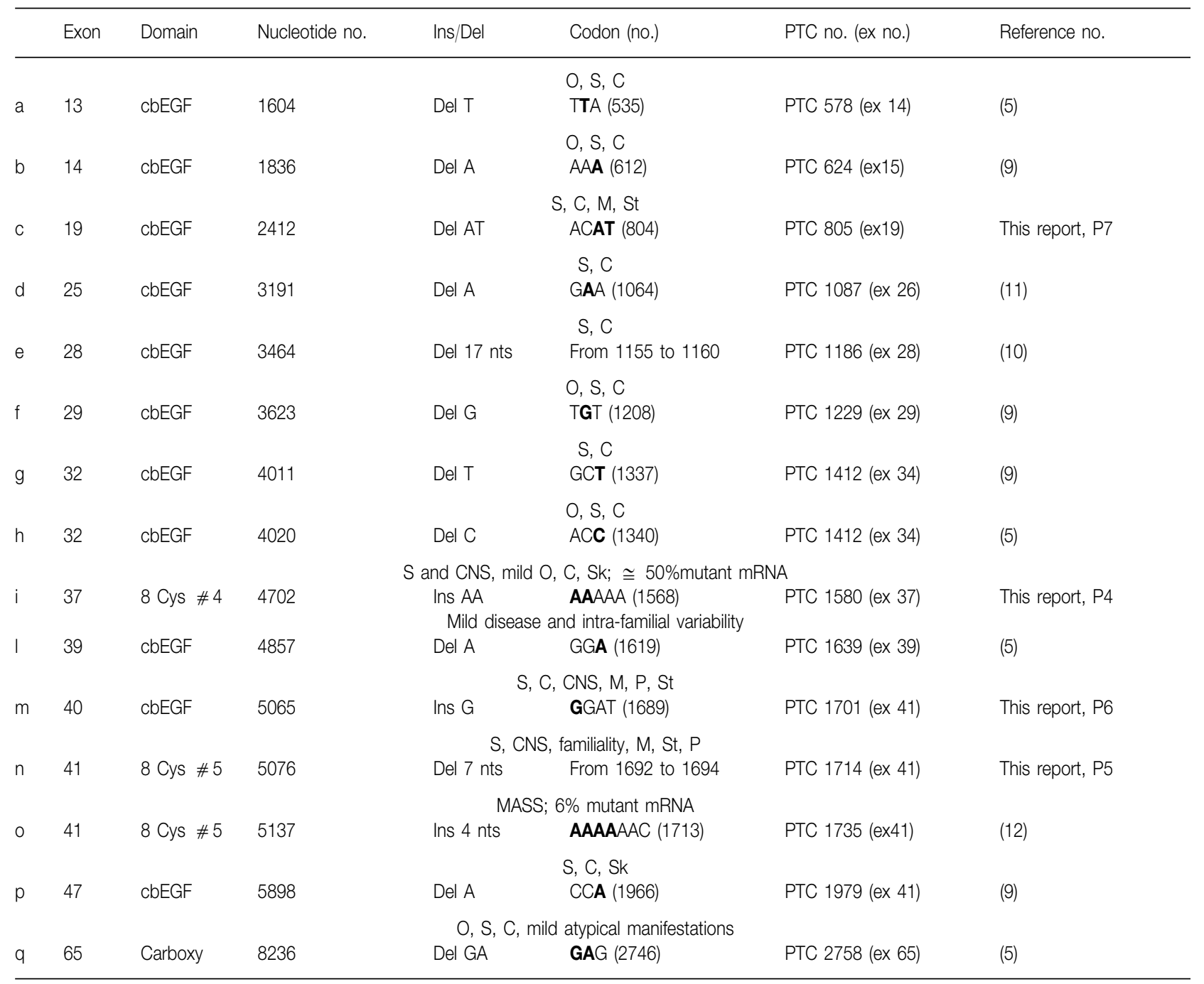

Small insertion/deletion frameshift mutation ( $<20 \mathrm{nts}$ ) creating premature termination codons (PTCs) in FBN1 gene are reported. $\mathbf{S}=$ skeleton; $\mathbf{C}=$ cardiovascular; $\mathbf{C N S}=$ central nervous system; $\mathbf{P}=$ pneumothorax; $\mathbf{S k}=$ skin; $\mathbf{M}=$ myopia; $\mathbf{S t}=$ striae. 
skeletal systems with an arched palate, mild pectus excavatum, joint hypermobility and striae atrophicae, mild myopia, with no alteration of the aortic root. Moreover, dural ectasia was excluded by MRI of the lumbo-sacral region.

One allele of P6 exon 40 displayed the insertion of a $\mathrm{G}$ between nucleotides 5064 and 5065 which also occurs in a stretch of Gs inside a trinucleotide TGG.

P7 is a 27-year-old Caucasian female with major criteria in the skeletal and cardiovascular system. She underwent surgery at the age of 12 for a severe scoliosis and presented with an arm span wider than her height, bilateral valgus elbow, arachnodactyly, positive wrist and thumb signs and bilateral pes equinus. She also had aortic root dilatation at the sinuses of Valsalva $\left(2.5 \mathrm{~cm} / \mathrm{m}^{2}\right.$ body surface area), and mild mitral and tricuspid valve prolapse. She had moderate bilateral myopia. Striae atrophicae localized at the glutei, not related to weight loss, were present. MRI of the lumbosacral region was not performed. No family history of MFS was reported although the grandfather, on the father's side, died at age 65 of sudden death.

One allele of P7 exon 19 presented with deletion of 2 nts (AT) corresponding to nucleotides 2412 and 2413. The deleted dinucleotide is positioned between a $\mathrm{C}$ and a TGA.

P4 is a 43-year-old Caucasian female with cardiovascular, ocular and major skeletal manifestations of MFS, without a family history of MFS although her father died of a thoracic aortic aneurysm (TAA). She presented with a reduced upperto-lower segment ratio. Her face was long and narrow with a high and narrow palate and retrognathia. Her eyes were deeply set. Pectus excavatum and severe scoliosis of the lumbar spine was present; she also had a vertebral hernia between L5 and S1 discs. Arachnodactyly, joint hyperextensibility, positive thumb and wrist signs were also present. The patient was asymptomatic with respect to the cardiovascular system. Auscultatory findings were unrevealing. Echocardiography showed localized aortic dilatation $\left(2.6 \mathrm{~cm} / \mathrm{m}^{2}\right.$ body surface area; n.v. $1.2-2.1 \mathrm{~cm} / \mathrm{m}^{2}$ ) of Valsalva sinuses. The sizes of ascending aorta, arch and descending aorta were normal. Valvular cusps also had an apparently normal morphology and there was no evidence of regurgitation. The mitral valve, however, was thickened and late systolic posterior bulging of the anterior leaflet was present. Ventricles and atria sizes were normal. Another major trait was the presence of a meningocele (MRI of grade 3 ).
Among the minor traits were: myopia, diagnosed at the age of 12 , representing the only ocular involvement. Hyperextensibility, with abdominal striae not associated with rapid weight change, varicose veins and tendency to ecchymoses were also evident.

One allele of P4 exon $37 F B N 1$ gene showed an insertion of two adenines between nucleotides 4701 and 4702. The insertion occurs in a stretch of As and is flanked by a pentanucleotide repeat (CTGGG).

P5 was a 29-year-old Caucasian female with severe skeletal manifestations. Her mother, affected by MFS, underwent surgery for De Bakey type III dissecting aneurysm (27) and died at the age of 42 of sudden death. A living sister (26 years old) is probably affected by MFS; she refused to come to hospital for a visit. P5 had pectus carinatum and excavatum of moderate/high severity; reduced upper-to-lower segment ratio; scoliosis $>20^{\circ}$, thumb and wrist signs. Among the minor manifestations were arachnodactyly, a moderately long and narrow face, a high and narrow palate with dental crowding, joint dislocation and shoulder hyperextensibility. Skin striae and atrophic scars were seen. Myopia was pronounced. She developed a spontaneous pneumothorax at 13 years of age.

Echocardiography detected a normal diameter of the aortic root $\left(1.72 \mathrm{~cm} / \mathrm{m}^{2}\right.$ body surface area $)$ but showed findings consistent with floppy mitral and tricuspid valve diagnosis. In fact, the leaflets of the tricuspid valve were thickened and redundant revealing a mild regurgitation. The mitral valve was thicker and more redundant than the tricuspid valve and there was also a marked elongation of the cordae tendineae. Late systolic posterior bulging into the left atrium of the anterior leaflet with regurgitation was present. The size of the right and left atria and ventricles were within normal limits. There were no signs of systolic or diastolic dysfunction. Late systolic click and murmur were audible. ECG revealed $\mathrm{T}$ wave inversions in the inferior limb leads and Holter monitoring showed sporadic premature ventricular beats. Lumbo-sacral MRI revealed a dural alteration of grade 2 .

An allele of P5 exon 41 had a 7 nts deletion (AAGTTTG) from nucleotides 5076 to 5082. The deletion occurs immediately after an AAG trinucleotide and before a TG dinucleotide repeat.

All the frameshift mutations caused a PTC (4/ $48,8.3 \%)$. The other 44 characterized mutations were missense $32 / 48(64.6 \%)$, nonsense $5 / 48$ (10.4\%), splicing 6/48 (12.5\%) and samesense $1 / 48$ $(2.1 \%)$. The latter is a single nucleotide substitution 


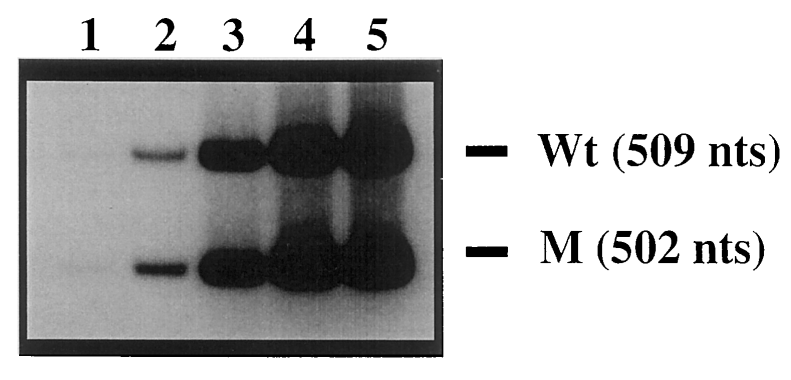

Fig. 1. Relative amount of the normal (509 nt) and mutant (502 nt) FBN1 mRNA from P5 fibroblasts. Hot RT-PCR was performed with a $\gamma^{33}$-P-ATP-labeled sense primer. The reactions were stopped at 17, 20, 23, 26 and 29 cycles (lanes 1-5). An aliquot ( 2 out of $25 \mu \mathrm{l}$ ) was loaded on an $8 \%$ polyacrylamide gel. The separated alleles were visualized by autoradiography and analyzed by densitometric scanning.

affecting the third position of a codon that does not change the aminoacid. This mutation has been demonstrated to cause exon skipping (26).

\section{mRNA quantification}

Semi-quantitative RT-PCR for quantifying the mutant mRNA was carried out in P5, the only patient from whom a skin biopsy was obtained. The ratio between the normal and mutant allele, measured densitometrically at various numbers of cycles, was approximately 1 (Fig. 1).

\section{Population screening}

In 100 randomly selected healthy subjects and 66 MFS patients, the four novel mutations were excluded by heteroduplex analysis (data not shown).

\section{DNA polymorphisms}

During heteroduplex analysis of the $65 F B N 1$ exons of the 4 patients, we detected two intronic sequence polymorphisms, both already described by Nijbroek and coworkers (5): an exon $28+15$ TTTTA del and an exon $41-14 \mathrm{~T}$ ins (data not shown).

\section{Discussion}

The nature and frequency of FBN1 mutations among our patients are in accordance with those in the literature with 11 of 137 FBN1 mutations causing a frameshift (i.e., $8 \%$ of the total mutations). Our 4 Italian patients all had a frameshift mutation in exons 37 and 41 (eight cysteine \# 3 and \#4 domains) and in exons 19 and 40 (cbEGF \# 8 and cbEGF \# 24 domains), respectively, representing $8.3 \%(4 / 48)$ of mutations in the Italian Marfan patients. These mutations appear to be novel.
All missense mutations in $F B N 1$ gene affecting cysteines important for the correct EGF-like domain structure or involving amino acids important for the calcium-binding act as dominant negative. The mutated monomers from one allele assemble with the normal monomers from the other allele creating abnormal multimers (12). These mutations usually correspond to a severe/moderate Marfan phenotype.

The frameshift mutations that create premature stop codons also act as dominant negative but display a highly variable clinical phenotype, from severe to mild (12). Therefore, the severity of the genotype is also directly related to the quantitative expression of the mutant allele and to the percentage of truncated proteins incorporated in the microfibrils $(5,28)$. The clinical variability is also evident in our patients. P4 and P5 present with major involvement of the skeletal and the central nervous system (dural ectasia). P4 carries the second mutation so far described in exon 37. Molecular analysis of the first mutation revealed a $G \rightarrow T$ transversion in the splice donor site of intron 37 that activates a cryptic site inside exon 37 producing a 15 amino acid deletion and a substitution of a Gly-Ser with an Ala.

It is noteworthy that in P4 the dinucleotide insertion at the protein level is close to the Arg-GlyAsp (RGD) sequence, a common cell recognition site which is present in a single copy in FBN1 (8). Furthermore, the frameshift deletion causes the formation of a PTC.

P5 has a $7 \mathrm{nts}$ frameshift deletion in exon 41 . Another mutation in exon 41, the 4 nts (TTCA) tandem insertion between nucleotides 5137 and 5138 causing a frameshift and a premature TGA stop codon (nucleotides 5199-5201) (Fig. 2) was described in a patient with a MASS phenotype (12). The patient described by Dietz and coworkers

\section{EXON 41}

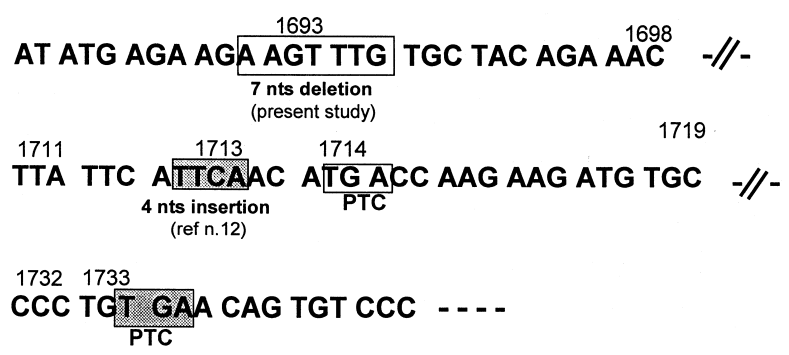

Fig. 2. Seven nucleotide deletion (white boxes) and $4 \mathrm{nt}$ insertion (grey boxes) with their corresponding PTCs in a Marfan patient and in a MASS patient, respectively, inside $F B N 1$ exon 41 . 
(12) carried a de novo mutation and presented with mitral valve prolapse, extreme dolichostenomelia, early myopia and striae distensae. Aortic root dimension, standardized to the body surface area, was at the upper normal limit. By comparing the clinical phenotype of the two patients with the same kind of mutation (frameshift quantitative mutation) in the same exon (exon 41) creating a premature stop codon 57 nts from each other (Fig. 2), P5 has similar, yet more severe, clinical stigmas. Interestingly, the two patients display a different percentage of mutant mRNA which is about $6 \%$ in the MASS patient (12) and 50\% in the Marfan patient (present study, Fig. 1). This quantitative difference can account, at least in part, for the variable phenotype in the frameshift mutants.

P6 is a classic Marfan patient carrying a deletion of the last nucleotide of exon 40 or the first nucleotide of intron 40. Considering that RT-RNA analysis of peripheral blood lymphocytes shows that intron 40 is spliced correctly (data not shown), we can classify the insertion as a frameshift mutation that causes the formation of a PTC in the same exon. P6 displays the most severe phenotype of all our patients suggesting, also in this case, the possibility of a highly expressed mutant allele.

In an attempt to make a phenotype-genotype correlation, we noticed that patients P4 and P5, who had mutations affecting 8 cysteine domains \# 4 and \# 5, respectively, had little or mild aortic involvement while $\mathrm{P} 6$, carrying a mutation in the cbEGF \# 24 domain, developed a TAA and an early AAA.

Only two patients have so far been reported to carry mutations in exon 41 and, for one of these, we do not have enough clinical data to make a comparison although he/she seems to present the involvement of the same apparatuses described in Dietz's patient $(9,12)$.

As for mutations in exon 40, there is only one case reported by Dietz and coworkers (30). Interestingly, in the only two mutations affecting the flanking exon 39 (cbEGF \# 23 domain), one is a frameshift mutation (5). This patient is a familial case with high clinical intra-familial variability. The father not only presents with thoracic aortic root aneurysm, but also underwent AAA surgery as a young adult. He was also the only one to carry ectopia lentis.

Insertion/deletion mutations below $20 \mathrm{nts}$ of DNA in a gene coding region may be explained by a) the presence of direct repeats flanking the insertion or of stretches of single bases which favor slippage mispairings at the replication fork and b) the presence of inverted repeats or symmetric elements which facilitate the formation of secondary- structure intermediates $(13,14)$. Up to now, three out of the $15 F B N 1$ frameshift mutations are insertions, two of which are made up of 1 and 2 nts, respectively (present study), while the other is made up of $4 \mathrm{nts}$ (12). On the other hand, most deletions $(n=8)$ are a result of the loss of $1 \mathrm{nt}$, and the remaining mutations are a result of the loss of $2(\mathrm{n}=2), 7(\mathrm{n}=1)$ and $17(\mathrm{n}=1) \mathrm{nts}$, respectively $((5,9-12)$; this report; Table 1$)$.

We reviewed all the frameshift insertion/deletion mutations $<20$ nts causing PTC so far described in FBN1 gene. They are all flanked by direct repeats or by stretches of single nucleotides and agree with the consensus sequences reported (TGA/GA/GG/TA/C), and are therefore a result of a mechanism of slipped mispairing $(13,14)$.

In conclusion, we suggest that frameshift mutations, when compared with missense mutations altering the structure or the function of a single domain, can produce a highly variable phenotype depending on the quantitative mRNA expression of the mutant allele. Unfortunately, in many cases we were not able to compare the clinical phenotypes of our patients with those reported in the literature, the latter not being circumstantially related. We believe, in fact, that a more detailed clinical description is necessary in order to make a genotype-phenotype correlation between patients described by other groups.

\section{Acknowledgements}

We thank Prof. Francesco Amaldi for stimulating discussion and Mary Lo Ponte for revising the English manuscript.

\section{References}

1. Dietz HC, Pyeritz RE. Mutations in the human gene for fibrillin-1 (FBN1) in the Marfan syndrome and related disorders. Hum Mol Genet 1995: 4: 1799-1809.

2. Pyeritz RE. The Marfan syndrome. In: Royce PM, Steinmann B, eds. Connective Tissue and its Heritable Disorders: Molecular, Genetic and Medical aspects. New York: Wiley-Liss, 1993: 437-468.

3. De Paepe A et al. Revised diagnostic criteria for the Marfan syndrome. Am J Med Genet 1996: 62: 417-426.

4. Tsipouras $P$ et al. Genetic linkage of the Marfan syndrome, ectopia lentis and congenital contractural arachnodactyly to the fibrillin genes on chromosomes 15 and 5: the International Marfan Syndrome Collaborative Study. N Engl J Med 1994: 326: 905-909.

5. Nijbroek $G$ et al. Fifteen novel FBN1 mutations causing Marfan syndrome detected by heteroduplex analysis of genomic amplicons. Am J Hum Genet 1995: 57: 8-21.

6. Sakai LY et al. Fibrillin, a new $350-\mathrm{kD}$ glycoprotein, is a component of extracellular microfibrils. J Cell Biol 1986: 103: 2499-2509.

7. Gibson MA et al. The protein components of the 12nanometer microfibrils of elastic and nonelastic tissues. J Biol Chem 1989: 264: 4590-4598. 
8. Pereira L et al. Genomic organization of the sequence coding for fibrillin, the defective product in Marfan syndrome. Hum Mol Genet 1994: 2: 961-968.

9. Collod-Béroud $\mathrm{G}$ et al. Marfan database (third edition): new mutations and new routines for the software. Nucleic Acids Res 1998: 26: 229-233.

10. Tynan K et al. Mutation screening of complete fibrillin-1 coding sequence: report of five new mutations, including two in 8-cysteine domains. Hum Mol Genet 1993: 2: 1813 1821.

11. Wang $M$ et al. Three novel fibrillin mutations in exon 25 and 27:classic versus neonatal Marfan. Hum Mut 1997: 9: 359-362.

12. Dietz $\mathrm{HC}$ et al. Four novel FBN1 mutations: significance for mutant transcript level and EGF-like domain calcium binding in the pathogenesis of Marfan syndrome. Genomics 1993: 17: 468-475.

13. Cooper DN, Krawczak M. Mechanisms of insertional mutagenesis in human genes causing genetic diseases. Hum Genet 1991: 87: 409-415.

14. Krawczak M, Cooper DN. Gene deletions causing human genetic disease: mechanisms of mutagenesis and the role of the local DNA sequence environment. Hum Genet 1991: 86: $425-441$.

15. Fattori $\mathrm{R}$ et al. Importance of dural ectasia in phenotypic assessment of Marfan's syndrome. Lancet 1999: 354 (9182): 910-913.

16. Sambrook J et al. Molecular Cloning. A Laboratory Manual. New York, NY: Cold Spring Harbor Laboratory Press, 1989.

17. Ganguly A et al. Conformation-sensitive gel electrophoresis for rapid detection of single-base differences in doublestranded PCR products and DNA fragments: evidence for solvent-induced bends in DNA heteroduplexes. Proc Natl Acad Sci USA 1993: 90: 10325-10329.

18. Ganguly A, Prockop DJ. Detection of mismatched bases in double stranded DNA by gel electrophoresis. Electrophoresis 1995: 16: 1830-1835.

19. Pepe $G$ et al. A major involvement of the cardiovascular system in patients affected by Marfan syndrome: novel mutations in fibrillin-1 gene. J Mol Cell Cardiol 1997: 29: 1877-1884.

20. Pepe G. A highly polymorphic (ACT)n VNTR (Variable Nucleotide of Tandem Repeats) locus inside intron 12 of COL1A2, one of the two genes involved in dominant Osteogenesis Imperfecta. Hum Mutat 1993: 2: 300-305.

21. Orita $M$ et al. Rapid and sensitive detection of point mutations and DNA polymorphisms using the polymerase chain reaction. Genomics 1989: 5: 874-879.

22. Attanasio $\mathbf{M}$ et al. Cytokine gene expression in human LPS- and IFN- $\gamma$-stimulated mononuclear cells is inhibited by heparin. Thromb Haemostas 1998: 79: 959-962.

23. Pepe $G$ et al. A heterozygous splice site mutation in COL6A1 leading to an in-frame deletion of the al (VI) collagen chain in an Italian family affected by Bethlem myopathy, B B R C 1999: 258: 802-807.

24. Araki A, Sako Y. Determination of free and total homocysteine in human plasma by high-performance liquid chromatography with fluorescence detection. J Chromatogr 1987: 422: 43-52.

25. Morse RP et al. Diagnosis and management of infantile Marfan syndrome. Pediatrics 1990: 86: 888-895.

26. Liu W, Qian C, Franke U. Silent mutation induces exon skipping of fibrillin-1 gene in Marfan syndrome. Nat Genet 1997: 16: 328-329.

27. DeBakey ME et al. Dissection and dissecting aneurysms of the aorta: twenty-year follow-up of five hundred twentyseven patients treated surgically. Surgery 1982: 92: 1118 1134.

28. Karttunen $\mathrm{L}$ et al. Two novel fibrillin-1 mutations resulting in premature termination codons but in different mutant transcript levels and clinical phenotypes. Hum Mutat 1998: 1 (Suppl): S34-S37.

29. McGrory J, Cole WG. Alternative splicing of exon 37 of FBN1 deletes part of an "eight cysteine" domain resulting in the Marfan syndrome. Clin Genet 1999: 55: 118-121.

30. Dietz $\mathrm{HC}$ et al. Clustering of fibrillin 1 (FBN1) missense mutations in Marfan syndrome patients at cysteine residues in EGF-like domains. Hum Mutat 1992: 1: 366374. 